\title{
Polyploidy in the Pancreas of the Normal and Diabetic Mutant Mouse
}

\author{
M. G. Ehrie and F. J. Swartz \\ Univ. of Louisville School of Medicine, Dept. of Anatomy, Health Sciences Center, Louisville, Kentucky, U.S.A.
}

Summary. The DNA content of endocrine and exocrine pancreatic nuclei of normal C57BL/KsJ and diabetic mutant $\mathrm{C} 57 \mathrm{BL} / \mathrm{Ks}-\mathrm{db} / \mathrm{db}$ mice was measured by Feulgen microdensitometry. The exocrine and endocrine pancreatic nuclei of the 4-week-old normal, 12-week-old normal, and 4-week-old (prehyperglycaemic) diabetic mutant mice contained diploid and tetraploid cells, while the 12-week-old (established hyperglycaemic) mutant contained diploid, tetraploid, and octaploid nuclei. The polyploidy in the endocrine pancreas of all these mice was confined to the B-cells, while the A-cells were always diploid.

Key words: Pancreatic islets in mice, genotypes: C57BL/KsJ and C57BL/Ks-db/db, light microscopy, B-cells, nuclear volume classes, polyploidy, diabetes.

In 1965 , a diabetic mutant was discovered among inbred C57BL/KsJ mice. Publications followed from the Jackson Laboratory documenting some unique characteristics of these mice $[1,2]$. Briefly, this mutant is an expression of a single autosomal recessive gene (db) with complete penetrance in homozygous mice $(\mathrm{db} / \mathrm{db})$. Their diabetic syndrome is characterized by early obesity at 3 to 4 weeks of age, with increased levels of plasma immunoreactive insulin, followed at 5 to 6 weeks of age by hyperglycaemia, glycosuria, higher levels of plasma insulin, and a rapid deposition of fat. As the syndrome progresses, the plasma insulin level begins to fall, the hyperglycaemia becomes more pronounced, and rapid weight loss occurs prior to death at 4 to 6 months of age. The islets of Langerhans exhibit pathological changes that reflect the onset of diabetes. The principal changes are a progressive degranulation of the B-cells, proliferation of pancreatic ducts around and within the islets as an attempt at islet cell neogenesis and eventual degeneration of B-cells.

Recently, Like and Chick have studied the islets of these C57 diabetic mice using both the light and electron microscopy [3-5]. Their radioautographic studies, and the conclusions drawn from them, were of particular interest to us, since we have described the occurrence of diploid, tetraploid and octaploid B-cells in the islets of Langerhans of the normal human pancreas [6]. Because the C57 diabetic mutant is being used as an experimental model for diabetes mellitus in man, we have elected to investigate the occurrence of polyploidy (defined here as multiple DNA classes) in the normal (C57BL/KsJ) mouse, and in the diabetic mutant (C57BL/Ks-db/db), as an attempt to understand further the pathogenesis of diabetes mellitus, as well as the occurrence and significance of polyploidy in both normal and diseased cells.

\section{Materials and Methods}

A total of 8 diabetic mutant $(\mathrm{C} 57 \mathrm{BL} / \mathrm{Ks}-\mathrm{db} / \mathrm{db})$ and 8 normal littermate (C57BL/KsJ) male mice, from the Jackson Laboratory colony, were studied. All mice were killed by decapitation and the pancreata fixed for $7 \mathrm{~h}$ in Zenker-formol. Briefly, our approach, previously described in detail [6], involves establishment of the relationship between nuclear DNA content (by Feulgen microdensitometry) and nuclear volume, and then the use of nuclear volume alone to determine the level of polyploidy. Due to technical problems, also described in our prior paper, DNA values are given as extinctions (optical densities) and are not indicative of the total DNA content of a particular nucleus. These values, which are proportional to the amounts of dye in cylindrical "plugs" measured through cut nuclei, are thus not comparable from one section to the next, due mainly to variations in section thickness. They do, however, establish accurately the occurrence of multiple amounts of DNA among nuclei in a single section.

Once a DNA-volume relationship is confirmed on Feulgen-stained sections, simple observation of tissues stained by Gomori's modification of the Mallory- 

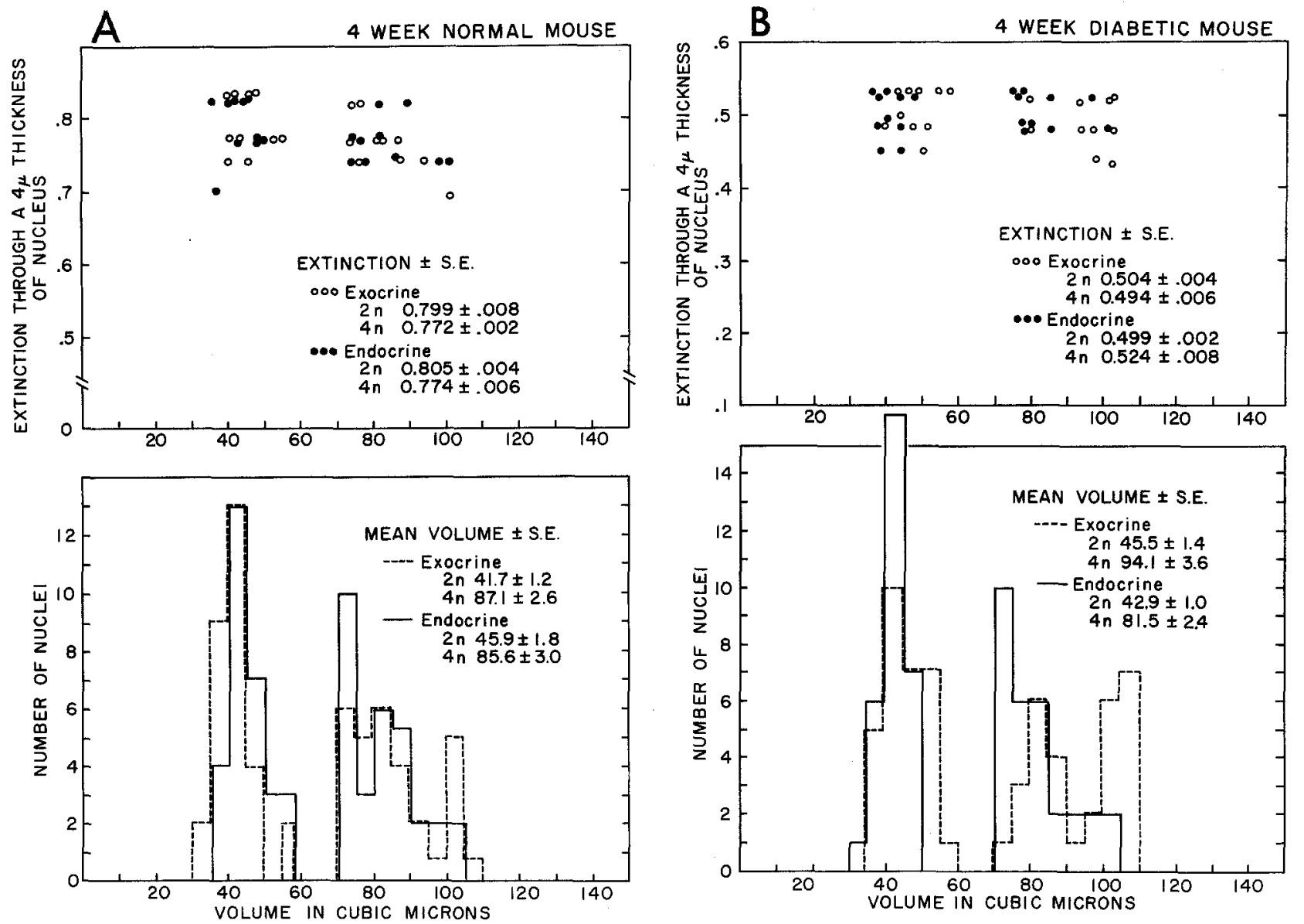

Fig. 1 A-B. Feulgen DNA extinction values correlated with nuclear volume (top), and nuclear volume determinations (bottom) of pancreatic nuclei of: A. normal 4-week-old C57BL/KsJ mouse; B. Diabetic 4-week-old C57BL/Ks-db/db mutant

Heidenhain azan method [7] permits the assignment of various pancreatic cells to a polyploid class.

Based on the classification of Chick and Like [5] we have, in the first of the two studies reported here, looked at 2 four week old prehyperglycaemic diabetic mice (4-6 weeks old, BG $120 \mathrm{mg} / \mathrm{ml}$ ), 2 twelve week established hyperglycaemic mice (8-20 weeks old, markedly obese, BG $200-400 \mathrm{mg} / 100 \mathrm{ml}$ ) and two normal littermate controls in each group. Detailed DNA-nuclear volume correlations were made on one animal in each group; visual confirmation of nuclear volumes were made on the second animal in each group; once confirmed by quantitative means, the presence of nuclear volume classes is readily apparent to the viewer. To verify these results, an identical study was repeated at a later date, on another group of C57 diabetic and normal mice, using only the volume and staining criteria.

\section{Results}

Table 1 shows the genotypes, ages, body weights, and tail lengths of the 16 mice studied.
For each of the four Group I animals studied in detail, DNA-volume correlations and numbers of nuclei in each polyploid class are shown in Fig. 1. All the specimens demonstrate distinct nuclear volume classes and, for each animal, extinction values are the same, indicating that the concentration of DNA is constant from one nuclear volume class to the next. Thus the DNA content of a pancreatic nucleus is proportional to its volume.

\section{Normal Mice}

Both the islets and the exocrine pancreas of 4 and 12 week normal mice (Fig. 1, A, C) show the presence of $2 n$ (diploid) and $4 n$ (tetraploid) nuclear classes. In the islets, the differentially stained sections showed, as in the human, that polyploidy is limited to B-cells. A number of binucleate exocrine cells were observed, but no mitotic figures were seen in either the islets or the exocrine tissue of these two age groups. These results were confirmed visually in the remaining 2 animals of Group I and in the four comparable animals of Group II. 

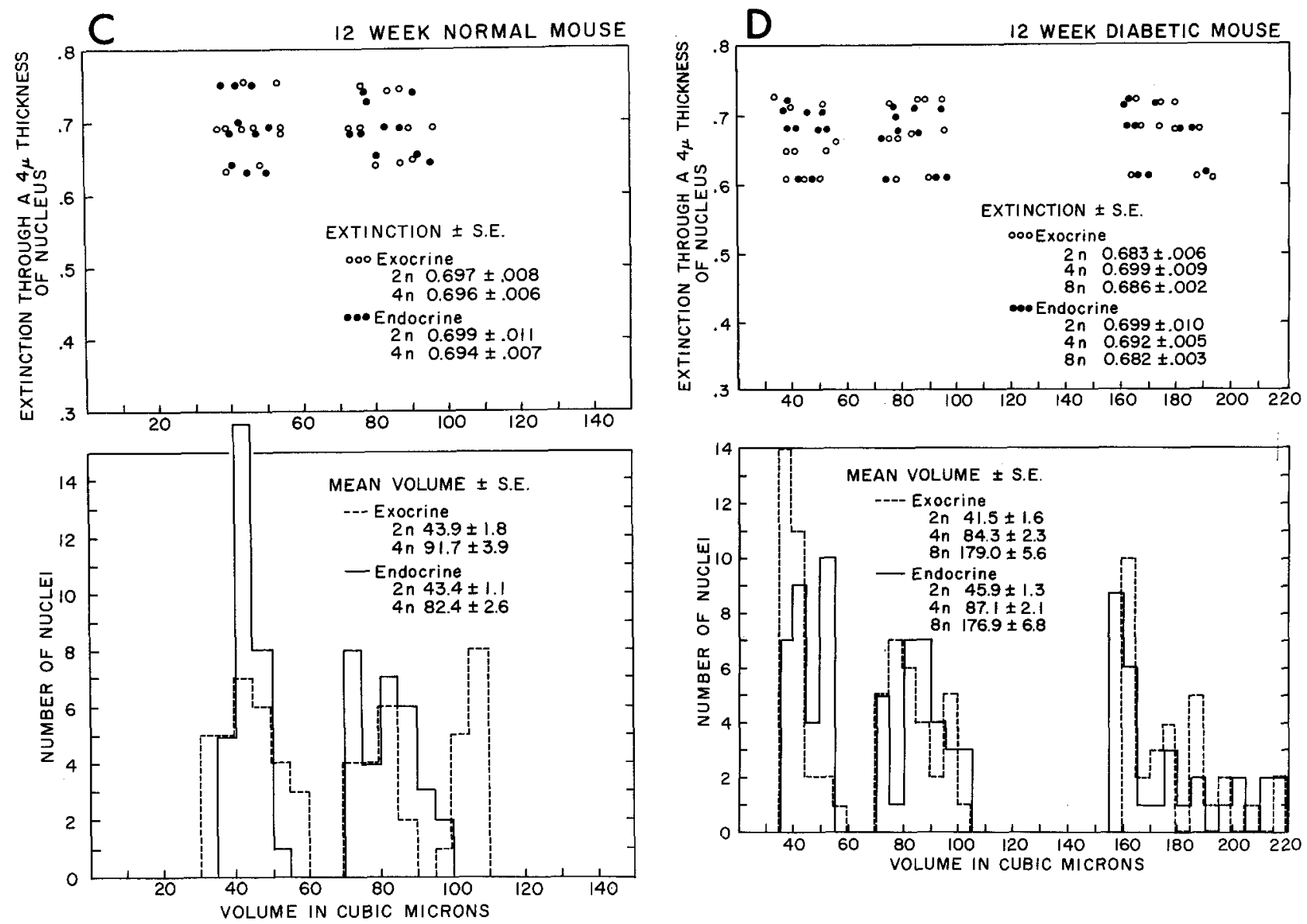

Fig. 1 C-D. Feulgen DNA extinction values correlated with nuclear volume (top), and nuclear volume determinations (bottom) of pancreatic nuclei of: C. normal 12-week-old C57BL/KsJ mouse; D. diabetic 12-week-old C57BL/Ks-db/db mutant

\section{Diabetic Mice}

In the prehyperglycaemic mice (Fig. 1, B), the same nuclear picture was observed as in the 4 and 12 week normal mice (Fig. 1, A). Islet polyploidy (tetraploidy) was again limited to B-cells. In the established hyperglycaemic mice (Fig. 1, D), however, the islets contained diploid, tetraploid, and octaploid B-cells, while A-cells remained diploid. Thus, a new class of islet nuclei (octaploid) appeared in the 12 week diabetic mice. Interestingly, a parallel increase in polyploidy of exocrine nuclei also emerged (Fig. 1, D). Although no mitotic figures were observed in the exocrine pancreas of the diabetic mice, mitoses were seen in the islets of both 4 and 12 week diabetic mice. These results were confirmed visually in the remaining 2 animals of Group I, and in the four comparable animals of Group II.

\section{Discussion}

Our prior paper reported the existence of diploid, tetraploid and octaploid cell nuclei in the human islet of Langerhans, while all other islet and exocrine nuclei were diploid. In the present study, polyploidy limited to B-cells has again been observed in the islets of normal mice, but the degree of polyploidy is one less than that observed in the human B-cell and, additionally, tetraploid exocrine nuclei are present. In the older diabetic mouse studied here (12 week established hyperglycaemic) octaploid B-cells, as well as octaploid exocrine cells, appear. Although Swift [8] seems to have been the first to document the existence of polyploid nuclei in mouse pancreas, he neither mentioned the strain of mouse studied nor differentiated between exocrine and endocrine cells. He did report the presence of three nuclear DNA classes in normal mouse pancreas, so it is possible that a small percentage of octaploid B-cells was present in our animals but remained undetected, or that this class would have eventually emerged with age. In fact, a few octaploid B-cells were found during our ultrastructural study of normal 12 week mice [9].

The significance of polyploid B-cells, as derived from these studies at the light level, remains speculative. The appearance of octaploid nuclei in the dia- 
Table 1. Relationship of age, body weight and tail length in one and three month old normal and diabetic mice

\begin{tabular}{llll}
\hline Genotype & Age (days) & Body weight $(\mathrm{g})$ & Tail length $(\mathrm{cm})$ \\
\hline $\mathrm{db} / \mathrm{db}$ & 30 & 21.0 & 7.0 \\
$\mathrm{db} / \mathrm{db}$ & 30 & 21.1 & 7.0 \\
$\mathrm{db} / \mathrm{db}$ & 30 & 18.3 & 6.2 \\
$\mathrm{db} / \mathrm{db}$ & 30 & 16.3 & 6.1 \\
Normal & 30 & 12.1 & 7.0 \\
Normal & 30 & 14.0 & 7.0 \\
Normal & 30 & 14.5 & 6.3 \\
Normal & 30 & 13.8 & 6.2 \\
\hline db/db & 86 & 55.0 & 8.1 \\
db/db & 86 & 48.0 & 8.1 \\
db/db & 84 & 50.4 & 8.4 \\
db/db & 84 & 50.4 & 8.5 \\
Normal & 86 & 25.0 & 8.1 \\
Normal & 86 & 24.1 & 8.1 \\
Normal & 84 & 25.6 & 8.9 \\
Normal & 84 & 26.0 & 8.9 \\
\hline
\end{tabular}

betic mouse suggests that the islets respond to diabetogenic stress with an increase in polyploidy. If, as has been proposed previously, polyploid cells perform more efficiently than their diploid counterparts, then a polyploid B-cell might be expected to produce proportionately more insulin, or be more heavily granulated, than a diploid B-cell. The degree of B-cell granulation could not be determined from this work, but electron microscopic observations on polyploid B-cells have provided some insight into this question [9].

The presence of tetraploid exocrine cells in normal mouse pancreas, and their parallel increase in level of polyploidy in diabetic mice, poses an interesting problem. It is tempting to speculate that the exocrine pancreas might, in some way, also be stressed in the diabetic syndrome of these mice.

The increased body weight of the older diabetic mice suggests that the additional polyploidy seen might simply be due to an accelerated growth rate, representing only the premature appearance of an otherwise normal, growth related phenomenon. The data in Table 1, however, indicate that this is not necessarily so. Although the body weights of the older diabetic mice are considerably greater than those of their normal controls, their tail lengths are the same, suggesting that there is no general growth acceleration. Furthermore, Malaisse et al. [10] have shown that pancreatic weights of normal and diabetic mutant mice in any particular age group are not significantly different.

Finally, two points of caution seem to be pertinent. The first relates to the use of the diabetic mouse as a model for human diabetes. Here, the differing cytological picture in the mouse and human pancreas, particularly with respect to the exocrine tissue, suggests the possibility that the basic disease processes in human and mouse may not be strictly comparable. Secondly, the incorporation of tritiated thymidine into islet nuclear DNA has been offered as evidence for islet cell neogenesis $[3,4]$. Our observations suggest that at least some of the observed uptake of tritiated thymidine is due to polyploidy rather than cell division.

Acknowledgements. Supported by USPHS General Research Support Grant. This work was part of a thesis submitted by the senior author to the Department of Anatomy, Graduate School of the University of Louisville in partial fulfillment of the requirements for the degree of Master of Sciences.

\section{References}

1. Coleman, D. L., Hummel, K. P.: Studies with the mutation diabetes in the mouse. Diabetologia 3, 238-248 (1967)

2. Coleman, D. L., Hummel, K. P.: The mutation, diabetes, in the mouse. In: Diabetes (Proc. 6th Congr. Int. Diabetes Fdn, Stockh. 1967), Excerpta Medica Int. Congress Series (Amst.) No. 172, 813-820 (1969)

3. Like, A. A., Chick, W. L.: Studies in the diabetic mutant mouse. I. Light microscopy and radioautography of pancreatic islets. Diabetologia 6, 207-215 (1970)

4. Like, A. A., Chick, W. L.: Studies in the diabetic mutant mouse. II. Electron microscopy of pancreatic islets. Diabetologia 6 , 216-242 (1970)

5. Chick, W. L., Like, A. A.: Studies in the diabetic mutant mouse. III. Physiological factors associated with alterations in beta-cell proliferation. Diabetologia 6, 243-251 (1970)

6. Ehrie, M. G., Swartz, F. J.: Diploid, tetraploid and octaploid beta-cells in the islets of Langerhans of the normal human pancreas. Diabetes 23, 583-588 (1974)

7. Gomori, G.: Studies on the cells of the pancreatic islets. Anat. Rec. 74, 439-460 (1939)

8. Swift, H. H.: The desoxyribose nucleic acid content of animal nuclei. Physiol. Zool. 23, 169-198 (1950)

9. Bowen, R. E., Swartz, F. J.: The ultrastructure of polyploid beta-cells in the islets of normal mice. Diabetologia 12 , 171-180 (1976)

10. Malaisse, W. J., Malaisse-Lagae, F., Coleman, D. L.: Insulin secretion in mice with an hereditary diabetes. Proc. Soc. exp. Biol. (N.Y.) 129, 65-69 (1968)

Received: August 25, 1975 and in revised form: February 11, 1976

Dr. F. J. Swartz

Univ. of Louisville

School of Medicine

Dept. of Anatomy

Health Sciences Center

P. O. Box 1055

Louisville, Kentucky 40201

USA 